Tropical Journal of Pharmaceutical Research April 2012; 11 (2): 169-175

(c) Pharmacotherapy Group,

Faculty of Pharmacy, University of Benin, Benin City, 300001 Nigeria.

All rights reserved.

Available online at http://www.tjpr.org

Research Article

http://dx.doi.org/10.4314/tjpr.v11i2.1

\title{
Effect of Process Factors on the Properties of Doxycycline Nanovesicles
}

\author{
S Honary* and F Zahir \\ Mazandaran University of Medical Sciences, School of Pharmacy, Pharmaceutical Sciences Research Center, Sari,
} Iran

\begin{abstract}
Purpose: To develop and evaluation ascorbyl palmitate niosomes in order to achieve a transdermal and/or systemic nanocarier of doxycycline.

Methods: Vesicles were formed from ascorbyl palmitate in combination with cholesterol and a negatively charged lipid, dicetyl phosphate. Niosomes were prepared by film hydration method followed by sonication in which aqueous doxycycline solution (in phosphate buffered saline) was encapsulated in the aqueous regions of the vesicles. The vesicles were evaluated for entrapment and in vitro release as well as for their thermal properties and shape by ultraviolet spectroscopy (UV), differential scanning calorimetry (DSC) and scanning electron microscopy (SEM), respectively. The effect of process conditions - sonication time, $\mathrm{pH}$, hydration temperature and centrifuge speed - on niosome properties was investigated.

Results: DSC of pure lipids, vesicles dispersion and mixture of lipids confirmed the formation of niosomes. Other results show that $>90 \%$ of drug was entrapped in the vesicles and the vesicles were spherical in shape. Drug release from the vesicles was slow (<60\% after 8 h). Nanovesicle size was significantly $(p<0.05)$ affected by sonication time and hydration $\mathrm{pH}$. Although hydration temperature of $100{ }^{\circ} \mathrm{C}$ promoted the production of smaller vesicles, this temperature would likely cause drug degradation.

Conclusion: The use of ascorbyl palmitate along with cholesterol and a charge inducer (dicetyl phosphate) yielded vesicles that satisfactorily encapsulated doxycycline solution. The resulting system can be applied for the formulation of doxycycline niosomes.
\end{abstract}

Keywords: Doxycicline, Vesicles, Drug delivery, Ascorbyl palmitate, Niosomes. 


\section{INTRODUCTION}

Tetracycline manifests a broad safety profile. Doxycycline requires less frequent administration and is considered safer than tetracycline [1]. Doxycycline, a semi-synthetic derivative of oxytetracycline is a potent antibacterial drug, commonly used as doxycycline hyclate [2]. Doxycycline is more active than tetracycline against many bacterial species including the entrococci and various anaerobes, protozoa and plasmodium. It has a broad-spectrum of activity against a wide variety of microorganisms, has been successfully used in man for more than 40 years and is commonly employed in the therapy of certain respiratory, skin, soft tissue and genitourinary infections, intestinal amoebiasis, anthrax, acute acne and also in the prophylaxis of malaria [2-3].

Other actions of doxycycline include differential inhibition of the activity of members of the matrix metalloproteinase (MMP) family, inhibition of interleukin-1 synthesis, inhibition of activated B cell function, inhibition of nitrous oxide (NO) synthesis by lipopolysaccharide (LPS) activated macrophage, and inhibition of collagen synthesis by cultured chondrocytes. With varying degrees of success it has been used to treat patients with rheumatoid arthritis [4]. It inhibits collagen synthesis and collagenase activity in hypertrophic chondrocytes, slowing the process of collagen turnover [5]. As with other tetracyclines it is remarkably tissue-irritating when injected, and so a prolonged release preparation of doxycycline can reduce this undesirable side effect [6].

Designing new vehicles for old drugs is an important field of pharmaceutical research, since the severe collateral effects of a drug can reduce greatly its therapeutic benefits [7]. Niosomes are one of the colloidal drug carriers [8]. Preliminary studies indicate that niosomes behave in vivo like liposomes which are usually produced using phospholipids, prolonging the circulation of entrapped drug to alter its organ distribution and metabolic stability or to prolong the contact time of drug with the applied tissues in topical applications [8]. Niosome properties make them a versatile carrier that is suitable for different systemic and topical applications, the administration of anti-inflammatory drugs, non-invasive vaccines, and anticancer and anti-infective agents [9]. They offer several advantages over liposomes such as higher chemical stability, intrinsic skin penetration, and lower cost [10].

There are a few materials with amphiphilic moieties that are biologically active. Uchegbu et al have synthesized palmitoyl muramic acid and N-palmitoyl glucosamine to improve immuno- adjuvant effect and/or targeting to malignant tissues [11]. Niosomes are vesicles formed from non-ionic surfactants in the manner analogous to liposomes preparation. They are capable of entrapping small watersoluble solutes within an aqueous core and they have been proposed as possible alternatives to liposomes in drug delivery and targeting [12].

Ascorbic acid esters are amphiphilic and delineating studies about their surface active properties and applications have been reported [13-14]. Ascorbyl palmitate (AP) is an antioxidant used in both cosmetics and food industry. It has been shown that this material can protect human erythrocytes from oxidative damage [15].

The aim of this paper was to encapsulate sufficient quantities of doxycycline in vesicles made with ascorbyl palmitate and to evaluate the resulting niosomes as possible transdermal and/or systemic carriers for drug delivery.

\section{EXPERIMENTAL}

\section{Materials}

Ascorbyl acid-6-palmitate, dicetyl phosphate, doxycycline hydrocholoride, and dialysis 
tubing (cellulose membrane, $12400 \mathrm{Mw}$ cutoff) were obtained from Sigma Aldrich Chemical Company, Germany. Cholesterol was obtained from Applichem, Germany while chloroform and methanol (analytical grade) were purchased from Merck, Germany

\section{Preparation of vesicles}

A lipid mixture of ascorbyl palmitate (AP, 200 $\mu \mathrm{mol}$ ) and cholesterol (Ch) in varying molar ratios plus $10 \mathrm{~mol} \%$ of dicetyl phosphate (DCP)) was dissolved in $9 \mathrm{ml}$ chloroform and $1 \mathrm{ml}$ of methanol in a round bottom flask under nitrogen atmosphere. The solvent was evaporated under reduced pressure at $50 \stackrel{\circ}{\circ}$. The lipid film was dried in an evaporator and then hydrated with $10 \mathrm{ml}$ of phosphate buffered saline (PBS, $\mathrm{pH}=7.4$ ) containing doxycycline $(2 \mathrm{mg} / \mathrm{ml})$ maintained at the same temperature. The vesicles were sonicated for varying times, using an ultrasonicator (Citizen SW 1500, India). Formation of vesicles was confirmed by SEM photographs as well as observation of unsonicated vesicles by light microscope (Zeiss, Germany). The vesicles were stored in nitrogen-purged vials at room temperature.

\section{Characterization of vesicles}

The vesicles were variously characterized as follows.

\section{Entrapment}

The encapsulation efficiency of the niosome formulations were determined by centrifugation of $5 \mathrm{ml}$ samples at $24000 \mathrm{rpm}$ for 15 min (high speed centrifuge, Hermle 236 HK, Germany). Supernatant doxycycline concentration was assessed by UV-Vis spectrophotometer (V 630, Jasco, Japan) at $\lambda \max$ of $265 \mathrm{~nm}$. The size of any particles in the supernatant was measured to ensure no vesicles were remained in the supernatant. Phosphate buffered saline (PBS, $\mathrm{pH}=7.4$ ) was used as blank and all measurements were performed in triplicate. Doxycycline entrapment efficiency (DE) was calculated as in Eq 1.

$D E=100(T D-F D) / T D$

where TD is the total doxycycline used in the preparation of the vesicles and FD is the doxycycline content of the supernatant

\section{Assessment of size, characteristics and zeta potential of vesicles}

The size, size distribution and zeta potential of the vesicles were determined by zetasizer nanoparticle analyser (Zetasizer 3600, Malvern instruments, UK) at $25^{\circ} \mathrm{C}$ and a scattering angle of $90^{\circ}$.

\section{Differential scanning calorimetry (DSC) of vesicles}

Both the vesicle dispersion and anhydrous mixture of AP/Ch/DCP were studied by DSC method (Perkin Elmer, Germany) to understand the thermotropic properties and phase transition behaviour of the vesicles. Analysis was carried out in a conventional aluminium pan. The pans covered with aluminium lid, sealed and perforated, and then heated from 50 to $250^{\circ} \mathrm{C}$ at $10^{\circ} \mathrm{C} / \mathrm{min}$. An empty loosely covered aluminium pan was used as the reference.

\section{Evaluation of in vitro doxycycline release}

The vesicles were collected after centrifugation of samples at $10000 \mathrm{rpm}$ for 20 min (Hermle 236 HK, Germany). The supernatant solutions were used for in vitro doxycycline release study. In vitro release was determined using a vertical diffusion cell. Prior to the release study, extent smuch of the unentrapped doxycycline was removed by dialyzing the samples in PBS $(\mathrm{pH}=7.4)$ for one hour. Four millilitres of dialyzed vesicle preparation was subjected to release studies. The receptor cell was filled with PBS. Dialyzed vesicles or doxycycline solution (in PBS, $\mathrm{pH}=7.4$ ) was transferred to the donor cell (5 $\mathrm{ml}$ capacity). Three $\mathrm{ml}$ samples were 
withdrawn from the receptor cell at various intervals over a period of $24 \mathrm{~h}$. The withdrawn sample was replenished with an equivalent volume of PBS. The samples were analyzed spectrophotometrically at $265 \mathrm{~nm}$.

\section{Scanning electron microscopy of the vesicles (SEM)}

A scanning electron microscope (model 2360 Leo Oxford, England) was used to analyze the surface and shape characteristics of the particles after prior coating with gold.

\section{Statistical analysis}

The results were expressed as mean \pm SD . Student's t-test and one-way analysis of variance (ANOVA) were applied to check significant differences in drug release from different formulations. Differences were considered to be statistically significant at $p<$ 0.05 . Sigma Plot 5 was used as the software for the analysis.

\section{RESULTS}

Ascorbyl palmitate and cholesterol, in the presence of dicetyl phosphate, a negatively charged lipid, formed stable spherical vesicles. Formation of vesicles was confirmed by SEM photographs of the vesicles as Fig 1) as well as observation of unsonicated vesicles by light microscopy (450 $\mathrm{x}$ and $1500 \times$ magnification).

\section{Effect of various process parameters on nanovesicle properties}

The effect of composition of the lipid materials on vesicles size, zeta potential and entrapment efficiency of doxycycline is shown in Table 1. The properties of the doxycycline nanovesicles were affected by process conditions. The size of doxycycline vesicles significantly decreased $(p<0.05)$ from 304 $\mathrm{nm}(\mathrm{F} 1)$ to $185 \mathrm{~nm}$ (F7) when the rate of ultracentrifuge increased from 5000 to 23000 RPM. The results also show that increasing the temperature from 50 to $70^{\circ} \mathrm{C}$ in the hydration stage had no significant effect on vesicles size.

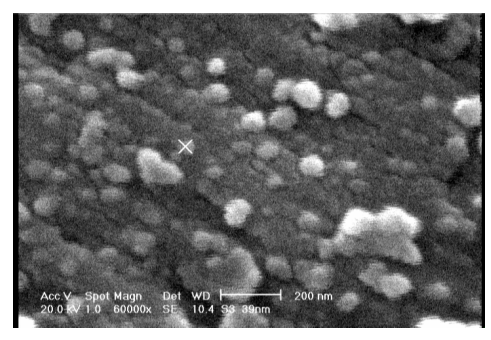

Figure 1: Scanning electron microphotographs of F5 niosomes

Although hydration temperature of $100{ }^{\circ} \mathrm{C}$ produced smaller vesicles, this temperature is high and could cause drug degradation.

The results (Fig 2) show that the size of the nanovesicles significantly decreased $(p<$ $0.05)$ from $275 \mathrm{~nm}$ (F8) to $183 \mathrm{~nm}$ (F10) and the vesicle size became more uniform when the sonication time was increased from 2 to $20 \mathrm{~min}$. Another factor is the hydration $\mathrm{pH}$ of the solution. Although decreasing the $\mathrm{pH}$ of hydration from 7.4 to 7 and 6.8 produced no significant difference in vesicle size, increase in $\mathrm{pH}$ from 7.4 to 8.0 produced smaller vesicles.

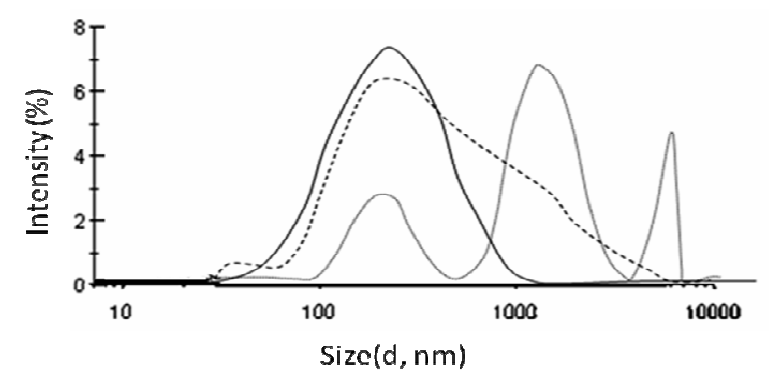

Fig 2: The size and size distribution profiles of vesicles obtained without sonication $\left({ }_{-}\right)$, with sonication for $2 \mathrm{~min}(\ldots .$.$) , and sonication for 20$ $\min (\ldots)$.

\section{DSC results}

DSC scans of anhydrous mixtures of lipid mixture in varying proportios nks could be related to dehydration, melting and

Trop J Pharm Res, April 2012;11 (2):172 
Table 1: The effect of different conditions on doxycycline vesicle

\begin{tabular}{|c|c|c|c|c|c|c|c|c|}
\hline Code & $\begin{array}{c}\text { Composition } \\
\text { (mol \%) }\end{array}$ & $\mathrm{pH}$ & $\begin{array}{c}\text { Temp } \\
\left({ }^{\circ} \mathrm{C}\right)\end{array}$ & $\begin{array}{l}\text { Rate of } \\
\text { ultracent } \\
\text { rifuge } \\
\text { RPM }\end{array}$ & $\begin{array}{l}\text { Time } \\
(\min )\end{array}$ & $\begin{array}{c}\text { Mean } \\
\text { size } \\
(\mathrm{nm})\end{array}$ & $\begin{array}{c}\text { Entrap } \\
(\%)\end{array}$ & $\begin{array}{l}\text { Zeta } \\
\text { pote } \\
\text { ntial } \\
(\mathrm{mV})\end{array}$ \\
\hline F1 & 45\%Ap:45\%Ch:10\%Dp & 7.4 & 50 & 5000 & 2 & 304 & 90 & -37 \\
\hline $\mathrm{F} 2$ & 45\%Ap:45\%Ch:10\%Dp & 7.4 & 50 & 10000 & 2 & 250 & 92 & -37 \\
\hline F3 & 45\%Ap:45\%Ch:10\%Dp & 7.4 & 50 & 15000 & 2 & 235 & 91 & -37 \\
\hline F4 & 45\%Ap:45\%Ch:10\%Dp & 7.4 & 50 & 20000 & 2 & 210 & 91.5 & -37 \\
\hline F5 & 45\%Ap:45\%Ch:10\%Dp & 7.4 & 50 & 23000 & 2 & 185 & 90.5 & -38 \\
\hline F6 & 45\%Ap:45\%Ch:10\%Dp & 7.4 & 70 & 10000 & 2 & 280 & 92 & -37 \\
\hline F7 & 45\%Ap:45\%Ch:10\%Dp & 7.4 & 100 & 10000 & 2 & 227 & 91.5 & -37 \\
\hline F8 & 45\%Ap:45\%Ch:10\%Dp & 7.4 & 50 & 10000 & - & 275 & 92 & -38 \\
\hline F9 & 45\%Ap:45\%Ch:10\%Dp & 7.4 & 50 & 10000 & 10 & 204 & 91 & -37 \\
\hline F10 & 45\%Ap:45\%Ch:10\%Dp & 7.4 & 50 & 10000 & 20 & 183 & 90.5 & -37 \\
\hline $\mathrm{F} 11$ & 45\%Ap:45\%Ch:10\%Dp & 7.4 & 50 & & 40 & 580 & 94 & -37 \\
\hline $\mathrm{F} 12$ & 45\%Ap:45\%Ch:10\%Dp & 7.4 & 50 & & 60 & 390 & 93 & -37 \\
\hline F13 & 45\%Ap:45\%Ch:10\%Dp & $\begin{array}{c}6.8 \\
7\end{array}$ & $\begin{array}{l}50 \\
50\end{array}$ & $\begin{array}{l}10000 \\
10000\end{array}$ & $\begin{array}{l}2 \\
2\end{array}$ & $\begin{array}{l}248 \\
246\end{array}$ & $\begin{array}{l}90.8 \\
91.5\end{array}$ & -38 \\
\hline F14 & 45\%Ap:45\%Ch:10\%Dp & 7 & 50 & 10000 & 2 & 246 & 91.5 & -38 \\
\hline F15 & 45\%Ap:45\%Ch:10\%Dp & 7.7 & 50 & 10000 & 2 & 219 & 92 & -39 \\
\hline F16 & 45\%Ap:45\%Ch:10\%Dp & 8 & 50 & 10000 & 2 & 222 & 91 & -39 \\
\hline F17 & 36\%Ap:54\%Ch:10\%Dp & 7.4 & 50 & 10000 & 2 & 432 & 94 & -38 \\
\hline F18 & 27\%Ap:63\%Ch:10\%Dp & 7.4 & 50 & 10000 & 2 & 325 & 93 & -38 \\
\hline F19 & 45\%Ap:45\%Ch:10\%Dp & 7.7 & 70 & 15000 & 20 & 181 & 91.5 & -39 \\
\hline
\end{tabular}

Key: $\mathrm{pH}=$ hydration $\mathrm{pH} ; \mathrm{Temp}=$ hydration temperature; $\mathrm{RPM}=$ centrifuge speed; Time = sonication time; Mean size = mean size of vesicles; Entrap = entrapment (\%); $Z P=$ zeta potential

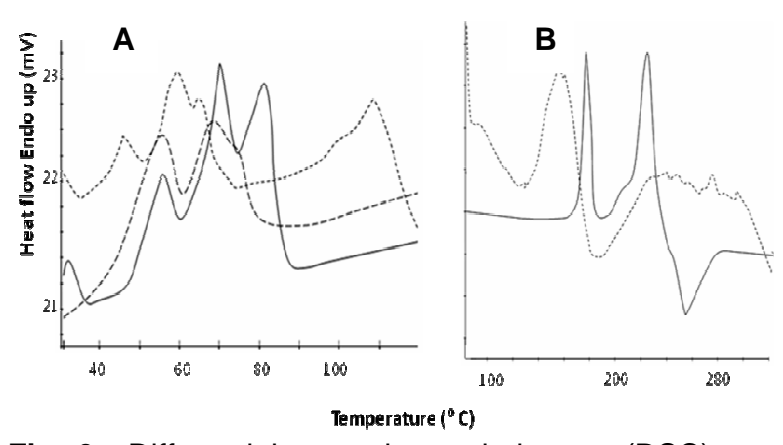

Fig 3: Differential scanning calorimetry (DSC) scans. (A) different $\mathrm{AP}, \mathrm{Ch}$ and $\mathrm{DCP}$ mixture: 45:45:10 (- - ) ), 36:54:10 (— ), and 27:63:10 $(--)$ ). (B) pure doxycycline $(-\cdot-)$, doxycycline vesicles ( ......... )

decomposition of docycycline, respectively (Fig. 3B). Doxycycline vesicle dispersion in PBS (Fig 3B) showed one main exothermic peak at $120^{\circ} \mathrm{C}$ followed by an endothermic peak at $140{ }^{\circ} \mathrm{C}$. Table 1 shows that variation in processing conditions did not show significant effect $(p<0.05)$ on entrapment and zeta potential of the vesicles

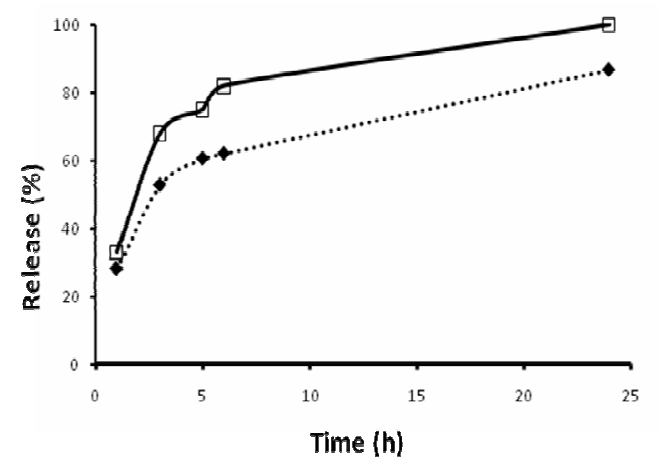

Fig 4: In vitro release profiles of doxycycline from vesicles ( $\bullet$ and doxycycline solution (口)

Fig 4 shows that the the release of drug from doxycycline vesicles was significantly slower $(p<0.05)$ than from the drug solution.

Trop J Pharm Res, April 2012;11 (2):173 


\section{DISCUSSION}

Based on the results obtained, the most important factor that influenced the size of the vesicles is the sonication time. Increasing ultra-sonication time to $20 \mathrm{~min}$ yielded more uniform and smaller vesicles. For example, the size of $5-\mathrm{Fu}$ niosomes decreased from 500 to $200 \mathrm{~nm}$ using this method. However, sonication of 5-Fu-loaded niosomes elicited a slight reduction of carrier capacity [9]. In the present work, sonication time had no significant effect on the entrapment efficiency of the vesicles.

Previously reported DSC scans of AP, Ch and DCP showed melting endotherms at 116 , 149 and $82{ }^{\circ} \mathrm{C}$, respectively [16]. DSC scans of various anhydrous fatty mixtures (AP, Ch and DCP) are shown in Fig.3A. The first peak is due to simple melting of eutectic mixture and this was followed by three other peaks; the first two peaks could related to mesospheric transition, or may be smectic or cholestric phases. It was shown earlier [16] that with increase in Ch concentration in lipid mixture, an increase in the area of the fourth peak was observed which could be related to pure $\mathrm{Ch}$. Increase in Ch concentration slightly decreased the main transition temperature. The liquid crystalline state is a prerequisite for the formation of vesicles[16]. The DSC results show evidence for the formation of liquid crystals with ascorbyl palmitate, cholesterol and DCP anhydrous mixture. The DSC thermogram of doxycycline pure powder shows two sharp endothermic peaks at 155 and $205{ }^{\circ} \mathrm{C}$, which was followed an exothermic peak at $230^{\circ} \mathrm{C}$. These peaks may be related to dehydration melting point and decomposition of docycycline at these temperatures, respectively. The vesicle dispersion of doxycicline in PBS (Fig 3B) showed one main exothermic peak at $120^{\circ} \mathrm{C}$, followed by an endothermic peak at $140^{\circ} \mathrm{C}$. This thermogram shows major differences from that of the pure doxycycline. Thus, the thermograms suggest vesicle formation and drug loading.
Flurbiprofen entrapment efficiency increased at acidic $\mathrm{pH}$ condition [10]. Decreasing $\mathrm{pH}$ could increase the proportion of the unionized species of an acidic drug, such as flurbiprofen,, which have higher partitioning to the bilayer lipid phase compared to the ionized species. However, a drug model such as doxycycline with different dissociation constants (3.5, 7.7 and 9.5) could show completely different results. The results demonstrated that changing $\mathrm{pH}$ in the range, 6.8 to 8 did not have significant effect on the size and entrapment of vesicles (table 1). Although increasing hydration temperature from 50 to $100{ }^{\circ} \mathrm{C}$ yielded smaller vesicles, it is not applicable owing to drug instability in high temperature. More than $90 \%$ of drug was entrapped in the vesicles in all formulations.

The zeta potential of formulations varied from -37 to $-39 \mathrm{mV}$. The stability of emulsion and colloids, according to DLVO electrostatic theory, is a balance between the attractive "Van der Waals" forces and the electrical repulsion because of the net surface charge. If the zeta potential falls below a certain level, the emulsion droplets or colloids will aggregate as a result of the attractive forces. Conversely, a high zeta potential (either positive or negative), typically more than 30 $\mathrm{mV}$, maintains a stable system [16].

In this kind of vesicles, polar ascorbyl head groups would project out of the vesicle surface, whereas palmitate chains would aggregate due to hydrophobic interaction. Overall, the free polar ascorbyl head groups, hydrophobicity/amphiphilicity and nanosize of this vesicle might enable it to neutralize reactive oxygen species of blood as well as produce much higher adhesion to biomembrans which might allow them to localize interacellularly than micro/macro size.

\section{CONCLUSION}

Ascorbyl palmitate formed vesicles in presence of cholesterol and charge inducer 
dicetyl phosphate, encapsulating Doxycycline solution. This system could be employed as a drug delivery system for the formulation of doxycycline niosomes. Vesicles were spherical and released their content slower than doxycycline solution. This technique reported may be useful in the production of injectable long-acting formulation of doxycycline.

\section{ACKNOWLEDGMENT}

This work was supported by the research section of Mazandaran University of Medical Sciences.

\section{REFERENCES}

1. Chaidemenos G. Tetracycline and Niacinamide in the Treatment of Blistering Skin Diseases. Clin Dermatol. 2001; 19: 781-785.

2. Vargas-Estrada D, Gracia-Mora J, Sumano $H$. Pharmacokinetic study of an injectable longacting parenteral formulation of doxycycline hyclate in calves. Res Vet Sci. 2007; 84: 477482.

3. Castro L, Sahagum A, Jose Diez M, Fernandez N, Sierra M, Garcia J. Pharmacokinetics of doxycycline in sheep after intravenous and oral administration. Vet J. 2009; 180: 389-395.

4. Smith VA, Cook SD. Doxycycline - a role in ocular surface repair. $\mathrm{Br} J$ Ophthalmol. 2004; 88: 619-625.

5. Karna E, Palka J, Wolczynski S. Doxycyclineinduced inhibition of prolidase activity in human skin fibroblasts and its involvement in impaired collagen biosynthesis. Eur $J$ Pharmacol. 2001; 430: 25-31.

6. Hannu T, Inman R, Granfors K, Leirisalo-Repo M. Reactive arthritis or post-infectious arthritis. Best Pract Res Clin Rheumatol. 2006; 20: 419-433.
7. Haerdi-Landerer $M$, Habermacher $J$, Wenger $B$, Suter M, Steiner A. Slow release antibiotics for treatment of septic arthritis in large animals. Vet J. 2009; 184:14-20.

8. Hu C, Rhodes D. Proniosomes: A Novel Drug Carrier Preparation. Int J Pharmaceut. 1999; 185: 23-35.

9. Paolino $D$, Cosco D, Muzzalupo R, Trapasso E, Picci N, Fresta M. Innovative bola-surfactant niosomes as topical delivery systems of 5fluorouracil for the treatment of skin cancer. Int J Pharmaceut. 2008; 353: 233-242.

10. Mokhtar M, Megrab N. Effect of some formulation parameters on flurbiprofen encapsulation and release rates of niosomes prepared from proniosomes. Int. J. Pharmaceut. 2008; 361: 104-111.

11. Uchegbu I. The biodistribution of novel 200-nm palmitory muramic acid vesicles. Int. J. Pharmceut. 1998; 162: 19-27.

12. Uchegbu I, Turton J, Double J, Florence A. Drug distribution and a pulmonary adverse effect of intraperitoneally administered doxorubicin niosomes in the mouse. Biopharm Drug Dispos 1994; 15:691-707.

13. Gopinath $D$, Ravi $D$, Rao BR, Apte $S$, Rambhau $D$. 1-O- Alkylglycerol vesicles (Algosomes): their formulation and characterization. Int. J. Pharmceut. 2002; 246:187-197.

14. Ross D, Mendiratta S, Qu Z, Cobb C, May J. Ascorbate 6-palomitate protects human erytrocytes from oxidative damage. Free Radical Bio Med. 1999; 26: 81-89.

15. Gopinath $D$, Ravi $D$, Rao BR, Apte $S$, Renuka $D$, Rambhau D. Ascorbyl palmitate vesicles (aspasomes): formulation, characterization and applications. Int. J. Pharmceut. 2004; 271: 95-113.

16. Honary S, Ebrahimi P, Tabbakhian M, Zahir F. Formulation and characterization of doxorubicin nanovesicles. J Vac Science Technology B 2009; 7:1573. 